\title{
Changes in Heart Rate Variability During Heartfulness Meditation: A Power Spectral Analysis Including the Residual Spectrum
}

\author{
Anne Léonard ${ }^{1}$, Serge Clément ${ }^{1}$, Cheng-Deng Kuo ${ }^{2,3 *}$ and Mario Manto ${ }^{1,4,5 *}$ \\ ${ }^{1}$ HELB Ilya Prigogine, Brussels, Belgium, ${ }^{2}$ Division of Chest Medicine, Department of Internal Medicine, Changhua Christian \\ Hospital, Changhua City, Taiwan, ${ }^{3}$ Department of Medical Research, Taipei Veterans General Hospital, Taipei, Taiwan, \\ ${ }^{4}$ Service de Neurologie, ULB CHU-Charleroi, Charleroi, Belgium, ${ }^{5}$ Service des Neurosciences, University of Mons, Mons, \\ Belgium
}

\section{OPEN ACCESS}

Edited by:

Shimon Rosenheck

Meir Medical Center, Israel

Reviewed by:

Osmar Antonio Centurion,

Universidad Nacional de

Asunción, Paraguay

Valeria Novelli,

Università Cattolica del Sacro

Cuore, Italy

*Correspondence:

Cheng-Deng Kuo

cdkuo23@gmail.com

Mario Manto

mmanto@ulb.ac.be

Specialty section

This article was submitted to

Cardiac Rhythmology,

a section of the journal

Frontiers in Cardiovascular Medicine

Received: 03 February 2019

Accepted: 29 April 2019

Published: 14 May 2019

Citation:

Léonard A, Clément S, Kuo C-D and Manto M (2019) Changes in Heart Rate Variability During Heartfulness

Meditation: A Power Spectral Analysis Including the Residual Spectrum. Front. Cardiovasc. Med. 6:62. doi: 10.3389/fcvm.2019.00062
Background: Meditation refers to a group of practices commonly proposed to treat stress-related conditions and improve overall wellness. In particular, meditation might exert beneficial actions on heart rate variability (HRV) by acting on autonomic tone with an increase in the vagal activity. The effects of heartfulness meditation (HM) on HRV remain poorly defined.

Methods: We investigated the effects of HM on HRV in a group of 26 healthy subjects. Subjects were regularly practicing this form of meditation on a daily basis. We assessed the HRV and residual HRV (rHRV) at rest and during meditation. We also used as control a period of respiratory rhythm imposed by an auditory signal, with the imposed breathing rhythm being identical to the spontaneous rhythm recorded during meditation.

Results: During deep meditation period, the standard deviation of RR intervals (SDRR), coefficient of variation of RR intervals $\left(C V_{R R}\right)$, and total power $(T P)$ were decreased while the low-frequency power (LFP), normalized LFP (nLFP), and normalized residual LFP (nrLFP) were increased as compared with those at rest, suggesting that the global vagal modulation was suppressed while the baroreflex was increased during deep medication. At the end of meditation, the LFP, residual LFP (rLFP), nLFP, nrLFP, low-/high-frequency power ratio (LHR), and residual LHR (rLHR) were increased while the residual very low-frequency power (rVLFP), normalized high-frequency power (nHFP), and normalized residual HFP (nrHFP) were decreased, as compared with those during paced breathing, suggesting that the vagal modulation was decreased while the sympathetic modulation was increased by deep meditation. During paced breathing period, the $\mathrm{SD}_{\mathrm{RR}}, \mathrm{CV}_{\mathrm{RR}}$, TP, LFP, rLFP, nLFP, nrLFP, LHR, and rLHR were decreased while nHFP and nrHFP were increased as compared with at rest, suggesting that paced breathing could suppress the sympathetic modulation and enhance the vagal modulation.

Conclusion: $\mathrm{HM}$ can induce a suppression of global vagal modulation and increased the sympathetic modulation and baroreflex. In addition, paced breathing can suppress the sympathetic modulation and enhance the vagal modulation. Unlike studies using other types of meditation, we did not identify evidence of increased vagal tone during HM.

Keywords: meditation, heartfulness, heart rate variability, power spectrum, residual spectrum, vagal, sympathetic, paced breathing 


\section{INTRODUCTION}

Heart rate variability (HRV) designates the continuous oscillations of successive RR intervals around the mean value $(1,2)$. The assessment of HRV by power spectral analysis allows extraction of the sympathetic and parasympathetic (vagal) modulations of heart rate (HR). Indeed, HRV results from the dynamic balance generated by the coactivation, co-inhibition, or reciprocal activation/inhibition of both the sympathetic and parasympathetic nervous systems (3). HRV is thus an indicator of regulation of the autonomous nervous system. This is particularly relevant in medicine since prolonged autonomic imbalance has been associated with a variety of somatic and mental disorders (4).

The terminology of meditation refers to a group of mental practices aiming to improve the overall wellness and to reduce stress. Meditation would be beneficial on a wide range of disorders (such as anxiety, depression, pain) and would exert positive effects upon HRV by increasing vagal tone. HRV was shown to be modulated during meditation and yoga practice (5, 6) with a positive effect on sympathovagal balance. Heartfulness meditation (HM) is a heart-based meditation practice aiming to reach a balance of mind (7). Participants redirect their mind toward the heart. Favorable effects on burnout and emotional wellness have been reported recently (7).

Although HM is used on a regular basis in many countries, its effects upon HRV are unknown. Our two goals were: (1) to evaluate whether HM increases HRV and residual HRV (rHRV), (2) to assess the effects of the respiration rhythm (spontaneously adopted by meditators during HM) upon HRV and rHRV, given the critical importance of respiratory patterns upon HRV spectra (8).

\section{METHODS}

The study was approved by the Ethical Committee of ULB Erasme (Reference 2016-521). All participating subjects signed a written informed consent after full explanation of the experimental procedures and before the experiment.

\section{Study Subjects}

We investigated the effects of $\mathrm{HM}$ on HRV and rHRV in a group of 26 healthy subjects (17F/9M; mean age \pm SD: $50.5 \pm 8.6$ years). Subjects were regularly practicing this form of meditation on a daily basis (mean duration of practice \pm SD: $13.1 \pm 7.4$ years). A total of 30 subjects were included in the study. Data were not analyzable in 4 subjects due to ECG recording artifacts. The results shown correspond to $n=26$ subjects.

\section{Inclusion and Exclusion Criteria}

Each participant used to practice HM for 1-h on a daily basis for a minimum of 4 years. All subjects were no smokers and not suffering from chronic alcoholism. They had no history of respiratory, cardiac and neurologic diseases such as stroke, brain tumor, cerebral malformation, epilepsy, neurosurgical intervention, migraine, cranial trauma, depression, Parkinson disease, tone disorders. None of them had an implantable pacemaker. They were taking no psychotropic drugs or narcotic substances.

Participants were asked not to consume alcohol within $12 \mathrm{~h}$, coffee/tea within $3 \mathrm{~h}$, and could not practice sports during the $12 \mathrm{~h}$ before the experiment.

\section{Questionnaires}

The following questionnaires were used:

- Freiburg Mindfulness Inventory (FMI): the French version has been used before the experimentation to measure dispositional mindfulness in daily activities. The score is related with years of experience.

- Mindfulness Attention Awareness Scale (MAAS)

Attention is a common characteristic of different types of meditation and seems to improve as meditators gain experience. Each subject was requested to fill in the questionnaire before the experimentation with aim of measuring the attention through subjective experiences.

- Meditation Depth Questionnaire (MEDEQ)

Following the 30 min-meditation ECG recording, a self-report to measure the depth of the meditative state was submitted to each subject. Adapted from the MEDEQ by Ott in 2001 and Thomas and Cohen (9), this questionnaire consists of a visual analog scale rated from 0 (no meditation) to 10 (deepest meditation state). We added 4 time marks in order to have the same reference on the ECG for the 30-min-meditation state and to compare the self-report data for each meditator and the ECG recording.

\section{Monitoring of Heart Rate and Respiration}

A non-invasive monitoring was performed using IWorx 214Data Recorder (iworx, Dover, USA). The device includes a duo channel bio potential amplifier. Wires are connected to collect ECG peripheral data using five electrodes positioned on wrists and ankles according to the recommendations of the Association for the Advancement of Medical Instrumentation. Another cable is connected to a respiration monitor RM-204 employing piezo technology to follow the relative depth and frequency of breathing. The measuring element is mounted in a belt that straps around the chest of the subject. Labscribe 2 software was used to collect and analyze data from ECG leads I and II and breathing.

\section{HRV Parameters}

HRV was computed from R-R intervals, as detailed previously $(1,2)$. The adjacent RR intervals were assessed for each study participant during 4 periods: during the rest period, during meditation (in the depth of meditation, at the end) and during the imposed respiratory rhythm period.

We assessed the HRV:

\section{(a) at rest}

(b1) during deep meditation

(b2) at the end of meditation

- (c) during "paced breathing": a control period with a respiratory rhythm imposed by an auditory signal. The imposed breathing rhythm was identical to the spontaneous rhythm recorded during meditation. 
The area under the curve of the spectral peaks within the frequencies range of $0.01-0.4,0.01-0.04,0.04-0.15$, and $0.15-$ $0.40 \mathrm{~Hz}$ were defined as the total power (TP), very low-frequency power (VLFP), low-frequency power (LFP), and high-frequency power (HFP), respectively.

In order to normalize VLFP, LFP, and HFP, we used the power within the frequency range of $0.01-0.4 \mathrm{~Hz}(1,2)$. The normalized VLFP (nVLFP $=$ VLFP/TP) is an index of vagal withdrawal, renin-angiotensin modulation, and thermoregulation (10). The normalized low-frequency power $(\mathrm{nLFP}=\mathrm{LFP} / \mathrm{TP})$ represents an index of combined sympathetic and vagal modulation (11) and an index of baroreflex $(12,13)$, and the normalized HFP $(\mathrm{nHFP}=\mathrm{HFP} / \mathrm{TP})$ corresponds to an index of vagal modulation. The low-/high-frequency power ratio $(\mathrm{LHR}=\mathrm{LFP} / \mathrm{HFP})$ is the index of sympathovagal balance.

\section{Respiratory Rate}

The respiratory rate was determined in breaths/min (bpm).

\section{Residual HRV}

The power spectrum of HRV was decomposed into a power-law function and a residual part of $\operatorname{HRV}(1,2)$,

$$
\mathrm{PSD}=\mathrm{F}_{\mathrm{rg}} \cdot \mathrm{rPSD}=10^{Y} \cdot \mathrm{Frq}^{\mathrm{s}} \cdot \mathrm{rPSD}
$$

where the PSD is the traditional power spectral density, $F_{r g}$ is the function of linear regression between $\log$ (PSD) and $\log$ (Frq) with slope "s" and Y-intercept " $Y$ " within the frequency range from $>0 \mathrm{~Hz}$ to the Nyquist frequency, "rg" stands for regression, Frq is the frequency, and rPSD is the residual PSD. The $F_{r g}$ is a power-law function of Frq with exponent "s" and normalization constant $10^{Y}$.

Similar to the definition of traditional HRV measures, the area-under-the-curve of the spectral peaks within the range of $0.01-0.4,0.01-0.04,0.04-0.15$, and $0.15-0.40 \mathrm{~Hz}$ in the residual power spectrum were defined as the residual total power (rTP), residual very low-frequency power (rVLFP), residual lowfrequency power (rLFP), and residual high-frequency power (rHFP), respectively. The normalized rVLFP (nrVLFP = $\mathrm{rVLFP} / \mathrm{rTP})$, normalized $\mathrm{rLFP}(\mathrm{nrLFP}=\mathrm{rLFP} / \mathrm{rTP})$, normalized rHFP $(\mathrm{nrHFP}=\mathrm{rHFP} / \mathrm{rTP})$, and residual low-/residual highfrequency power ratio $(\mathrm{rLHR}=\mathrm{rLFP} / \mathrm{rHFP})$ were defined in similar ways to those of traditional HRV measures.

\section{Statistical Procedures}

Statistical analysis was performed using Matlab and Sigmaplot ${ }^{\circledR}$ (Jandel Scientific, Germany). Descriptive statistics were computed. Mean, median, quartiles, and SD values were extracted. Statistical significance was set at 0.05.

Data normality was assessed using the Shapiro-Wilk test. To test the difference in ECG parameters between conditions, a non-parametric analysis of variance on ranks was made using a Friedman test. When Friedman test was positive, post-hoc analysis was done with a Wilcoxon rank test. Comparisons of respiratory rhythm at rest and during meditation periods were done with Student $t$-test.

\section{RESULTS}

\section{Effects of HM on HRV and rHRV}

A typical example of the effects of HM upon spectra of HRV for one subject is illustrated in Figure 1. HM caused a reduction of standard deviation of $\mathrm{RR}$ intervals $\left(\mathrm{SD}_{\mathrm{RR}}\right)$, coefficient of variation of RR intervals $\left(\mathrm{CV}_{\mathrm{RR}}\right)$, and total power (TP).

Table 1 shows that during deep meditation period b1, the $\mathrm{SD}_{\mathrm{RR}}, \mathrm{CV}_{\mathrm{RR}}$, and TP were significantly decreased as compared with those measures at rest a, and the LFP, nLFP, and nrLFP were significantly increased as compared with those measures during the control period $c$ when the breathing rhythm was paced in accordance with the spontaneous rhythm. At the end of meditation b2, the LFP, rLFP, nLFP, nrLFP, LHR, and rLHR were increased, while the rVLFP, nHFP, and nrHFP were decreased, as compared to the corresponding HRV and rHRV measures in the control period of paced breathing c. During the control period of paced breathing $\mathrm{c}$, the $\mathrm{SD}_{\mathrm{RR}}, \mathrm{CV}_{\mathrm{RR}}$, TP, LFP, rLFP, nLFP, nrLFP, LHR, and rLHR were decreased, while the nHFP and nrHFP were increased, as compared with the corresponding HRV and rHRV measures at rest a.

\section{Effects of HM on Respiratory Parameters}

The respiratory frequency was significantly higher $\left(p<10^{-5}\right.$ in b1 and $p=0.02$ in b2) during meditation when compared with rest condition (Figure 2) As illustrated on Figure 3, a significant positive linear relationship exists between the increase in the depth of meditation and the respiratory rate $(\rho=0.9685$, $p=0.02$ ). As shown on Figure 4, respiratory amplitude was significantly lower during meditation b1 and b2 when compared to rest condition $(p \leq 0.0006)$. Moreover, there is a weakly significant negative correlation between respiratory amplitude and the depth of meditation (Figure 5; $\rho=-0.3297, p=0.05$ ).

\section{DISCUSSION}

The goal of our study was to investigate the effect of HM on the autonomic nervous system and the potential influence of the breathing rhythm on the HRV and rHRV. We found a reduction of some HRV and rHRV measures during HM. Unlike studies using other types of meditation, we did not found an increased parasympathetic tone during $\mathrm{HM}$.

The decreases in $\mathrm{SD}_{\mathrm{RR}}, \mathrm{CV}_{\mathrm{RR}}$, and $\mathrm{TP}$ and the increases in LFP, nLFP, and nrLFP during deep meditation period as compared with those at rest suggested that the global vagal modulation was suppressed while the sympathetic modulations and baroreflex of the subjects were enhanced during deep medication. The increases in LFP, nLFP, and nrLFP during deep meditation period as compared with those during paced breathing suggested that deep meditation could increase both vagal and sympathetic modulations and baroreflex of the subjects. At the end of meditation, the increases in LFP, rLFP, nLFP, nrLFP, LHR, and rLHR and the decrease in rVLFP, nHFP, and nrHFP also suggested that vagal modulation was decreased while the sympathetic modulation and baroreflex were increased by deep meditation, as compared with paced breathing. 
HRV
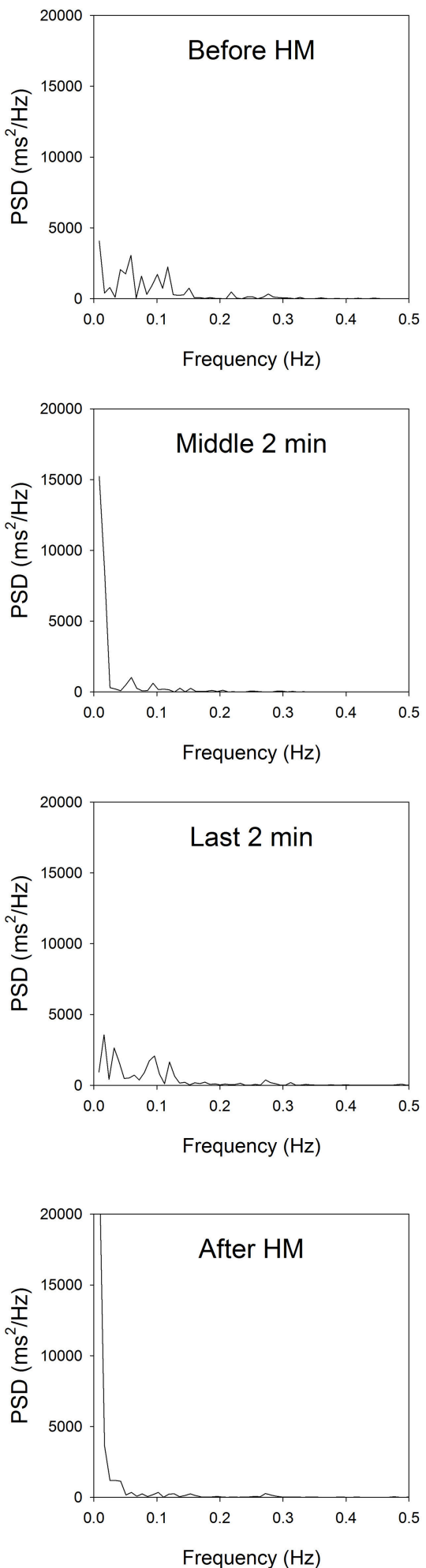

rHRV
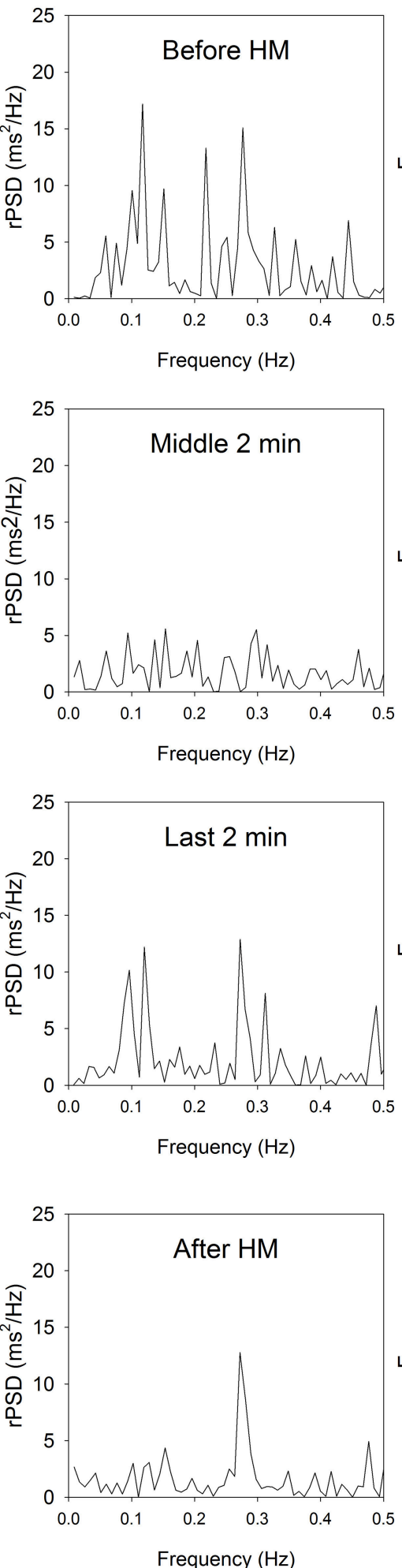

Power law function
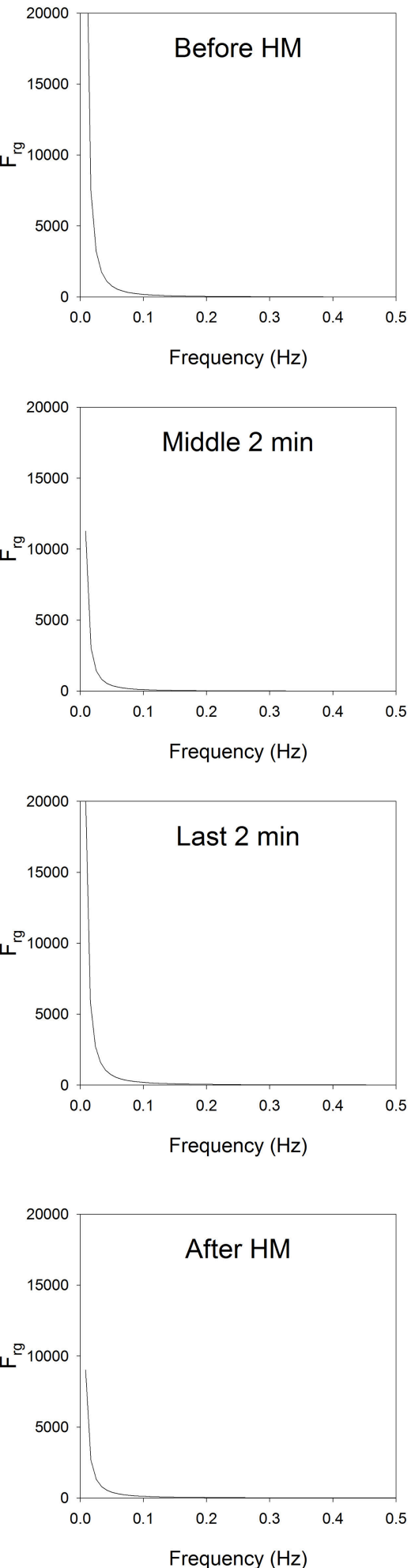

FIGURE 1 | HRV spectrum, residual HRV spectrum and the power law function of a representative participant. Top, before HM; middle upper panels, middle of HM; middle lower panels, last 2 min of HM; bottom panels, imposed breathing after HM. In all stage of HM and after HM, the very low-frequency part of the rHRV is reduced, the low-frequency part of $r \mathrm{HRV}$ is reduced, while the high-frequency part of the rHRV is enhanced. 
TABLE 1 | Comparison of HRV and rHRV measures among at rest (a), during deep medication (b1), at the end of medication (b2), and during the control period (c).

\begin{tabular}{|c|c|c|c|c|c|}
\hline & At rest (a) & Deep meditation (b1) & End of meditation (b2) & Control period (c) & $\boldsymbol{P}$ \\
\hline mRRI & 842 (754-916) & 833 (759-925) & $815(767-883)$ & 822 (760-899) & 0.361 \\
\hline $\mathrm{HR}$ & $71.3(65.5-79.5)$ & $72.1(64.9-79.0)$ & $73.6(68.0-78.2)$ & $73.0(66.7-78.9)$ & 0.351 \\
\hline $\mathrm{SD}_{\mathrm{RR}}$ & $28.1(21.3-42.9)^{\#}$ & $22.8(20.4-38.2)^{\star}$ & $31.0(18.9-40.2)$ & $24.0(19.5-32.5)^{\star}$ & 0.014 \\
\hline $\mathrm{CV}_{\mathrm{RR}}$ & $0.033(0.029-0.047)^{\#}$ & $0.031(0.026-0.042)^{\star}$ & $0.036(0.02-0.047)$ & $0.027(0.024-0.034)^{*}$ & 0.002 \\
\hline RMSSD & $20.2(11.6-32.7)$ & $19.2(12.0-33.4)$ & $18.6(12.2-28.3)$ & $18.3(12.3-27.4)$ & 0.302 \\
\hline TP & $390.2(223.1-885.9)^{\#}$ & $258.0(194.8-712.6)^{\star}$ & $467.4(174.3-793.8)$ & $279.0(180.2-511.7)^{\star}$ & 0.010 \\
\hline rTP & $1.224(0.935-1.689)$ & $1.360(1.083-1.641)$ & $1.313(0.982-1.652)$ & 1.417 (1.115-2.387) & 0.183 \\
\hline VLFP & $82.2(56.2-133.0)$ & $67.6(28.4-112.9)$ & $64.2(29.5-77.0)$ & $51.4(23.0-93.7)$ & 0.059 \\
\hline rVLFP & $0.028(0.016-0.045)$ & $0.033(0.016-0.052)$ & $0.020(0.011-0.032)^{\#}$ & $0.047(0.031-0.067)$ & $<0.001$ \\
\hline LFP & $144.1(77.7-317.5)^{\#}$ & $114.0(45.5-180.6)^{\#}$ & $134.0(61.7-347.7)^{\#}$ & $40.4(25.3-82.8)^{\star}$ & $<0.001$ \\
\hline rLFP & $0.375(0.204-0.538)^{\#}$ & $0.252(0.188-0.479)$ & $0.412(0.260-0.689)^{\#}$ & $0.187(0.154-0.259)^{\star}$ & $<0.001$ \\
\hline HFP & 83.7 (31.0-238.0) & 83.7 (45.7-244.3) & $60.8(31.3-137.6)$ & 74.2 (29.9-199.8) & 0.477 \\
\hline rHFP & $0.639(0.424-1.110)$ & $0.818(0.585-1.261)$ & $0.538(0.430-1.207)$ & 1.131 (0.665-1.778) & 0.057 \\
\hline nVLFP & $17.4(13.0-28.2)$ & $17.7(11.6-30.2)$ & $13.4(10.3-18.3)$ & 18.8 (9.8-28.2) & 0.270 \\
\hline nrVLFP & $1.86(1.08-5.36)$ & $2.21(0.99-4.80)$ & $1.54(0.74-3.43)$ & $3.18(1.09-5.22)$ & 0.073 \\
\hline nLFP & $38.0(18.4-3.6)^{\#}$ & $35.1(20.1-3.9)^{\#}$ & $44.7(20.6-4.0)^{\#}$ & $21.0(15.5-3.0)^{\star}$ & $<0.001$ \\
\hline nrLFP & $33.11(22.47-45.28)^{\#}$ & $23.06(16.74-38.53)^{\#}$ & $37.75(21.51-55.79)^{\#}$ & $13.59(9.30-25.48)^{\star}$ & $<0.001$ \\
\hline nHFP & $24.6(17.0-3.3)^{\#}$ & $29.6(19.2-3.8)$ & $25.4(0.2-4.0)^{\#}$ & $34.7(24.6-4.8)^{\star}$ & 0.017 \\
\hline nrHFP & $62.91(52.35-76.83)^{\#}$ & 73.66 (59.99-78.78) & $58.26(41.65-77.21)^{\#}$ & $83.19(65.61-88.81)^{\star}$ & $<0.001$ \\
\hline LHR & $1.87(0.72-3.99)^{\#}$ & $1.16(0.63-2.03)$ & $2.56(0.85-7.39)^{\#}$ & $0.61(0.29-1.92)^{\star}$ & $<0.001$ \\
\hline rLHR & $0.52(0.29-0.89)^{\#}$ & $0.31(0.21-0.64)$ & $0.65(0.28-1.38)^{\#}$ & $0.17(0.11-0.40)^{\star}$ & $<0.001$ \\
\hline
\end{tabular}

${ }^{*} P<0.05$ vs. at rest (a), ${ }^{\#} P<0.05$ vs. during the control period of paced breathing (c).

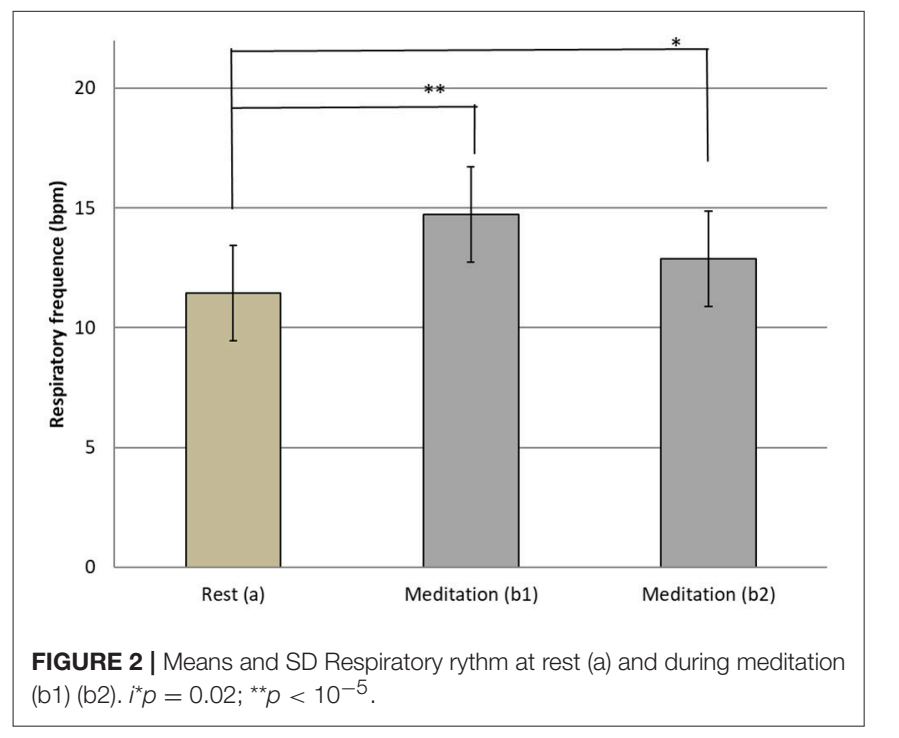

The effect of paced breathing on autonomic nervous modulation could also be observed by comparing the HRV and rHRV measures during paced breathing with those at rest. The decreases in $\mathrm{SD}_{\mathrm{RR}}, \mathrm{CV}_{\mathrm{RR}}$, TP, LFP, rLFP, nLFP, nrLFP, LHR, and rLHR and the increases in nHFP and nrHFP during paced breathing suggested that paced breathing could suppress the sympathetic modulation and baroreflex, and enhance the vagal modulation.

It is interesting to note that the effects of meditation on the HRV indices were very similar to the effects of meditation on

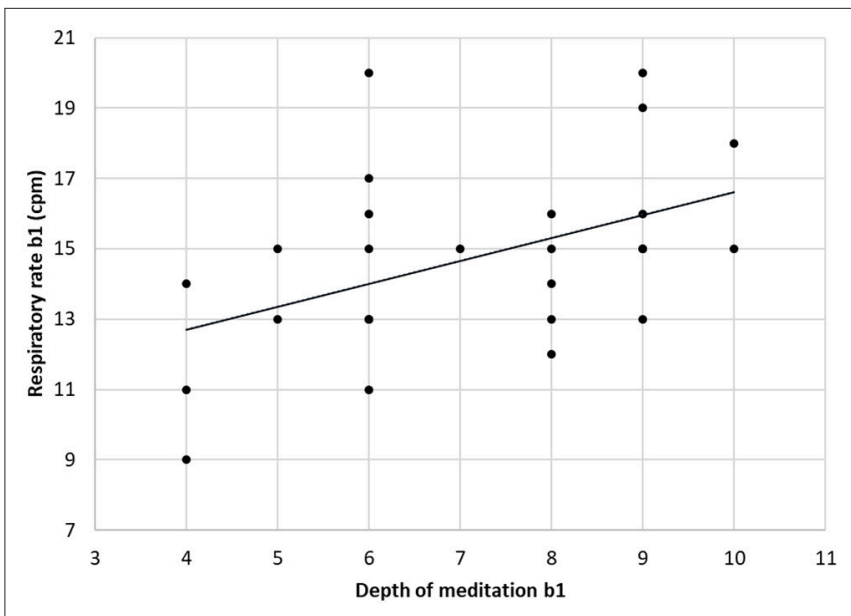

FIGURE 3 | Linear correlation between respiratory rate and depth of mediation $(\rho=0.9685, p=0.02)$.

the rHRV indices except the TP and rTP. This is comprehensible because the rTP is the residual total power after the removal of the power law function from the HRV spectrum. Despite of some studies highlighting the differences in respiratory rhythm at rest (14) linked with years of practice, we couldn't observe any correlation between experience and a reduction in respiratory rhythm at rest in our experiment.

In order to assess the changes that could be acquired by years of meditation practice, self-reported scale has been used such as in other studies (15-17). For this purpose, we used MAAS and 


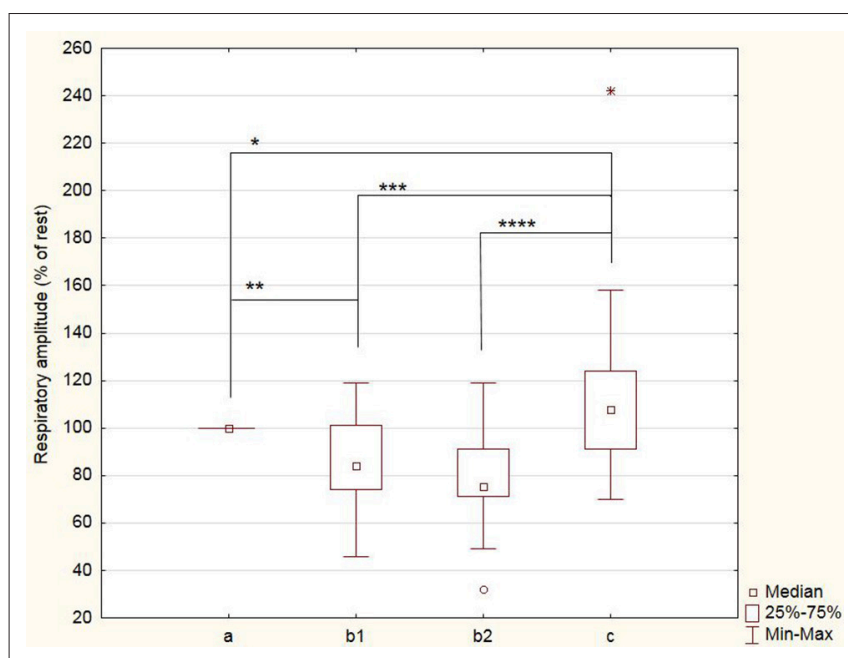

FIGURE 4 | Breathing amplitude during meditation (b1) and (b2) and during control period of paced breathing (c). Amplitude are expressed as percent of rest condition. ${ }^{\star} p=0.04 ;{ }^{\star \star} p=0.0006 ;{ }^{\star \star \star} p=0.0005 ;{ }^{\star \star \star \star} p=0.0002$.

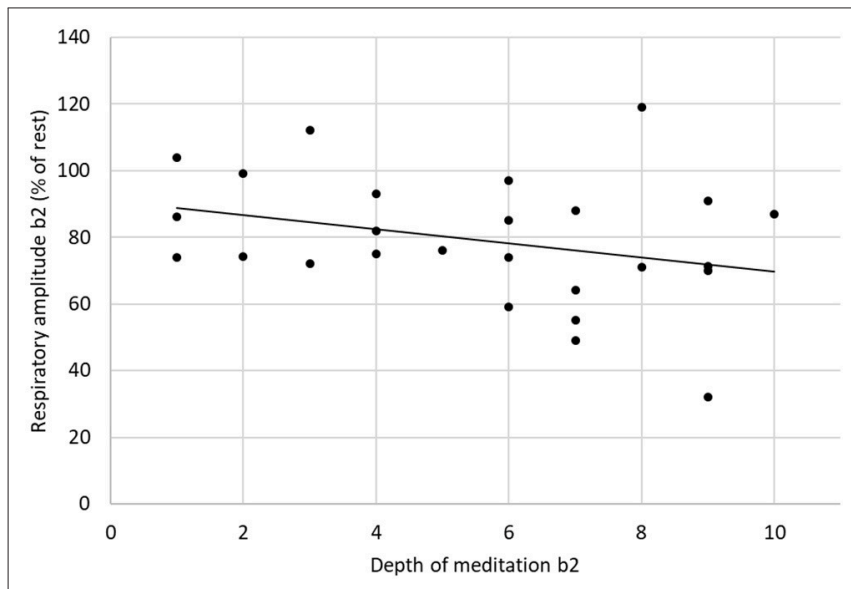

FIGURE 5 | Linear correlation between respiratory amplitude and depth of meditation $(\rho=-0.3297, p=0.05)$.

FMI to evaluate an improvement in attention capacity and in mindfulness. But the results didn't show any correlation between scores and years of experience.

Very few studies on meditation have assessed the depth of meditation. Yet the meditative state is a subjective state that could vary from one moment to another. The Meditation Depth Questionnaire (the sole questionnaire we found in the literature) adapted from Ott (18) and Thomas and Cohen (9) was submitted to the subject to ensure that the subject was well-immersed in meditation during the data recording. We selected experimented "meditators," who were aware of the quality of their meditation state. The Meditation Depth Questionnaire was submitted to the participants right after finishing the meditation practice. Our approach is similar to the pain scale used in a variety of medical and surgical settings to determine the severity, type, and duration of the pain. Similarly, we can quantify the meditation depth by scoring it from 1 to 10 , with 10 represents the most profound state of meditation. This is a limitation of our study because we rely on the feeling of the participant. Even if this tool is subjective, it allowed us to estimate the correlation between the meditation depth and the change in the breathing rate in subjects.

These data showed a significant linear correlation between the depth of meditation and the breathing rate. In other words, deeper is the meditative state and higher is the breathing rate. This correlation has, to our knowledge, not yet been observed in other studies.

We have shown that HM did not modify the heart rate but reduce HRV significantly $\left(\mathrm{SD}_{\mathrm{RR}}, \mathrm{CV}_{\mathrm{RR}}\right.$, TP, VLFP, LFP, HFP, LHR). If we look more closely at the results we have obtained, during meditation, an increase in HFnorm alone, which may reflect a modulation of the parasympathetic during meditation. But this increase is not sufficiently significant compared to the rest condition. While all the variables of HRV decrease during meditation, this increase could be explained, in part, by a very significant decrease in low frequencies (in absolute values and in normalized units). Thus, if there is a significant decrease in LF, the HFnorm can increase in an exaggerated way while the HF in the total spectrum decrease. This phenomenon has been already highlighted by Krygier et al. (17).

The use of variables in absolute values or in normalized units could also explain the disparity of the results obtained in terms of frequencies in studies to interpret an increase in parasympathetic tone during meditation. Indeed, some studies observe an increase in low frequencies (LF) interpreted as a modulation of the parasympathetic or sympathetic system or attributed to the degree of expertise of the subject or the task requested (19-21) or slow breathing rate (22). In contrast, other studies attributed the increase in HRV to an increase in normalized high frequencies (HF) together with a decrease in normalized LFs and interpreted as a predominance in vagal tone $(17,23,24)$. Supplementary Table 1 summarizes the results published in literature showing the discrepancy between the studies in connection with the types of meditation.

Besides the interpretation that each author can give to these variables, we can also observe that the breathing parameters and the type of meditation could explain the heterogeneity of the results in terms of frequencies. A study conducted in 2015 trying to answer to the question "Is meditation always relaxing?" showed that depending on the type of meditation, frequency variations are different. They concluded that observing-thoughts meditation and loving-kindness meditation leaded to an increased stimulation of the sympathetic system compared to breathing meditation (25).

Our finding showed that both conditions, "meditation" and "paced breathing" had an effect on reducing variables such as the standard deviation of $\mathrm{RR}$ intervals $\left(\mathrm{SD}_{\mathrm{RR}}\right)$, the coefficient of variation $\mathrm{RR}\left(\mathrm{CV}_{\mathrm{RR}}\right)$ and the total spectral power (TP). However, the effects of these two conditions cannot be distinguished statistically. So, it did not allow us for these variables to conclude to a meditation effect different from the respiration in terms of an overall decrease in HRV.

On the other hand, the statistically significant decrease in the low frequency frequencies (LF) (LFP, rLFP, nLFP, nrLFP) and 
the low-/high- frequency ratio (LHR) would be due solely to a breathing effect. The only statistically significant increase in the HRV spectrum was normalized high frequencies (HFnorm) which would also be solely due to an effect breathing.

Respiratory rhythm is often associated with psychological well-being and meditation seemed to lead to a decrease of it Wielgosz et al. (14), Nijjar et al. (23). In our study, the respiratory rhythm of subjects increased significantly during meditation compared to the rest condition. The breathing amplitude is another important data to be taken into account in this analysis. Indeed, compared to the "meditation" condition, the breathing amplitude in "paced breathing" condition is significantly higher. The subject thus has to breathe in a range and to a respiratory rate higher than the one he has at rest, so in a condition close to hyperventilation.

Posture and breathing may influence the HRV. The most determining factor in the amplification of the HRV is a slow and deep breathing rhythm (26). Rapid breathing or hyperventilation would impact on the HRV, but would induce an increase in the HFnorm, which could result from an increased tidal volume and respiration rate (27). Critchley et al. (28) examined the effects of hypoxia and slow breathing on the HRV, and assessed the neural substrates using fMRI. They noted during hypoxia a decrease in the HRV and a suppression of the baroreflex, as represented by the decrease in the LF. The authors found that dorsal medullary and pontine activity correlated positively with tidal volume and correlate inversely with heart rate. The activity in rostroventral medulla was correlated with blood pressure and HRV. We speculate that the modifications in these centers closely associated with sympathetic regulation may explain the impact of HM on sympathetic tone. A fMRI study combined with cardiovascular and respiratory measures should be performed to test this hypothesis. A study about the effects of relaxation and hyperventilation in anxious people, shows that relaxation does not really affect HFnorm and HRV. However, hyperventilation induces a decrease in HRV and an increase in HFnorm, such as our study (29).

\section{REFERENCES}

1. Kuo J, Kuo CD. Decomposition of heart rate variability spectrum into a power-law function and a residual spectrum. Front Cardiovasc Med. (2016) 3:16. doi: $10.3389 /$ fcvm.2016.00016

2. Jiang JS, Kor CT, Kuo DD, Lin CH, Chang CC, Chen GY, et al. Residual heart rate variability measures can better differentiate patients with acute myocardial infarction from patients with patent coronary artery. Ther Clin Risk Manag. (2018) 14:1923-31. doi: 10.2147/TCRM.S178734

3. Berntson GG, Bigger JT Jr, Eckberg DL, Grossman P, Kaufmann PG, Malik $\mathrm{M}$, et al. Heart rate variability: origins, methods, and interpretive caveats. Psychophysiology. (1997) 34:623-48. doi: 10.1111/j.1469-8986.1997.tb02140.x

4. Thayer JF, Ahs F, Fredrikson M, Sollers JJ III, Wager TD. A meta-analysis of heart rate variability and neuroimaging studies: implications for heart rate variability as a marker of stress and health. Neurosci Biobehav Rev. (2012) 36:747-56. doi: 10.1016/j.neubiorev.2011.11.009

5. Arya NK, Singh K, Malik A, Mehrotra R. Effect of heartfulness cleaning and meditation on heart rate variability. Indian Heart J. (2018) 70(Suppl. 3):S50-5. doi: 10.1016/j.ihj.2018.05.004

\section{CONCLUSION}

We found that HM could induce a suppression of global vagal modulation and increase the sympathetic modulation and baroreflex. In addition, imposed breathing rhythm could suppress the sympathetic modulation and enhance the vagal modulation. Unlike studies using other types of meditation, we did not identify evidence of increased vagal tone during HM. Our results suggest that the changes in breathing which occur during meditation influence HRV.

\section{ETHICS STATEMENT}

The study was approved by the Ethical Committee of ULB Erasme (Reference 2016-521). All participating subjects signed a written informed consent after full explanation of the experimental procedures and before the experiment.

\section{AUTHOR CONTRIBUTIONS}

AL, SC, and MM: design of the study. AL: data collection. AL, SC, C-DK, and MM: data analysis and drafting and approval of the final version.

\section{FUNDING}

$\mathrm{MM}$ is supported by the FNRS and the Fonds Erasme. C-DK is supported by a grant 106-CCH-IRP-100 from Changhua Christian Hospital, Changhua, Taiwan, and another grant V102C-058 from Taipei Veterans General Hospital, Taipei, Taiwan.

\section{SUPPLEMENTARY MATERIAL}

The Supplementary Material for this article can be found online at: https://www.frontiersin.org/articles/10.3389/fcvm. 2019.00062/full\#supplementary-material

6. Chu IH, Wu WL, Lin IM, Chang YK, Lin YJ, Yang PC. Effects of Yoga on heat rate variability and depressive symptoms in women: a randomized controlled trial. J Altern Complement Med. (2017) 23:310-6. doi: 10.1089/acm.20 16.0135

7. Thimmapuram J, Pargament R, Sibliss K, Grim R, Risques R, Toorens E. Effect of heartfulness meditation on burnout, emotional wellness, and telomere length in health care professionals. J Community Hosp Intern Med Perspect. (2017) 7:21-7. doi: 10.1080/20009666.2016.1270806

8. Beda A, Simpson DM, Carvalho NC, Carvalho AR. Low-frequency heart rate variability is related to the breath-to-breath variability in the respiratory pattern. Psychophysiology. (2014) 51:197-205. doi: 10.1111/psyp.12163

9. Thomas JW, Cohen M. A methodological review of meditation research. Front Psychiatry. (2014) 5:74. doi: 10.3389/fpsyt.2014.00074

10. Taylor JA, Carr DL, Myers CW, Eckberg DL. Mechanisms underlying verylow-frequency RR-interval oscillations in humans. Circulation. (1998) 98:547755. doi: 10.1161/01.CIR.98.6.547

11. Koizumi K, Terui N, Kollai M. Effect of cardiac vagal and sympathetic nerve activity on heart rate in rhythmic fluctuations. J Auton Nerv Syst. (1985) 12:51-9. doi: 10.1016/0165-1838(85)90065-7 
12. Moak JP, Goldstein DS, Eldadah BA, Saleem A, Holmes C, Pechnik $S$, et al. Supine low-frequency power of heart rate variability reflects baroreflex function, not cardiac sympathetic innervation. Cleve Clin J Med. (2009) 76(Suppl. 2):S51-59. doi: 10.3949/ccjm.76. s2.11

13. Rahman F, Pechnik S, Gross D, Sewell L, Goldstein DS. Low frequency power of heart rate variability reflects baroreflex function, not cardiac sympathetic innervation. Clin Auton Res. (2011) 21:133-41. doi: 10.1007/s10286-010-0098-y

14. Wielgosz J, Schuyler BS, Lutz A, Davidson RJ. Long-term mindfulness training is associated with reliable differences in resting respiration rate. Sci Rep. (2016) 6:27533. doi: $10.1038 /$ srep27533

15. Zeidan F, Emerson NM, Farris SR, Ray JN, Jung Y, McHaffie JG, et al. Mindfulness meditation-based pain relief employs different neural mechanisms than placebo and sham mindfulness meditation-induced analgesia. J Neurosci. (2015) 35:15307-25. doi: 10.1523/JNEUROSCI.2542-15.2015

16. Hauswald A, übelacker $T$, Leske $S$, Weisz $N$. What it means to be Zen: marked modulations of local and interareal synchronization during open monitoring meditation. Neuroimage. (2015) 108:265-73. doi: 10.1016/j.neuroimage.2014.12.065

17. Krygier JR, Heathers JA, Shahrestani S, Abbott M, Gross JJ, Kemp AH. Mindfulness meditation, well-being, and heart rate variability: a preliminary investigation into the impact of intensive Vipassana meditation. Int J Psychophysiol. (2013) 89:305-13. doi: 10.1016/j.ijpsycho.2013. 06.017

18. Ott U. The EEG and depth of meditation. I Meditation Med Res. (2001) 1:55-68.

19. Delgado-Pastor LC, Perakakis P, Subramanya P, Telles S, Vila J. Mindfulness (Vipassana) meditation: effects on P3b event-related potential and heart rate variability. Int J Psychophysiol. (2013) 90:207-14. doi: 10.1016/j.ijpsycho.2013.07.006

20. Phongsuphap S, Pongsupap Y, Chandanamattha P, Lursinsap C. Changes in heart rate variability during concentration meditation. Int J Cardiol. (2008) 130:481-4. doi: 10.1016/j.ijcard.2007.06.103

21. Peressutti C, Martín-González JM, García-Manso JM, Mesa D. Heart rate dynamics in different levels of Zen meditation. Int J Cardiol. (2010) 145:142-6. doi: 10.1016/j.ijcard.2009.06.058

22. Steinhubl SR, Wineinger NE, Patel S, Boeldt DL, Mackellar G, Porter V, et al. Cardiovascular and nervous system changes during meditation. Front Hum Neurosci. (2015) 9:145. doi: 10.3389/fnhum.2015.00145
23. Nijjar PS, Puppala VK, Dickinson O, Duval S, Duprez D, Kreitzer MJ, et al. Modulation of the autonomic nervous system assessed through heart rate variability by a mindfulness based stress reduction program. Int J Cardiol. (2014) 177:557-9. doi: 10.1016/j.ijcard.2014.08.116

24. $\mathrm{Wu} \mathrm{SD,} \mathrm{Lo} \mathrm{PC.} \mathrm{Inward-attention} \mathrm{meditation} \mathrm{increases} \mathrm{parasympathetic}$ activity: a study based on heart rate variability. Biomed Res Tokyo. (2008) 29:245-50. doi: 10.2220/biomedres.29.245

25. Lumma AL, Kok BE, Singer T. Is meditation always relaxing? Investigating heart rate, heart rate variability, experienced effort and likeability during training of three types of meditation. Int J Psychophysiol. (2015) 97:38-45. doi: 10.1016/j.ijpsycho.2015.04.017

26. Młyńczak M, Niewiadomski W, Cybulski G. Impact of breathing mechanics, body posture and physique on heart rate variability. In: Jabłoński R, Brezina T, editors. Advanced Mechatronics Solutions. Advances in Intelligent Systems and Computing. Vol. 393. Cham: Springer (2016). p. 111-6. doi: 10.1007/978-3-319-23923-1_16

27. Pöyhönen M, Syväoja S, Hartikainen J, Ruokonen E, Takala J. The effect of carbon dioxide, respiratory rate and tidal volume on human heart rate variability. Acta Anaesthesiol Scandin. (2004) 48:93-101. doi: 10.1111/j.1399-6576.2004.00272.x

28. Critchley HD, Nicotra A, Chiesa PA, Nagai Y, Gray MA, Minati L, et al. Slow breathing and hypoxic challenge: cardiorespiratory consequences and their central neural substrates. PLOS ONE. (2015) 10:e0127082. doi: 10.1371/journal.pone.0127082

29. Pittig A, Arch JJ, Lam CW, Craske MG. Heart rate and heart rate variability in panic, social anxiety, obsessive-compulsive, and generalized anxiety disorders at baseline and in response to relaxation and hyperventilation. Int J Psychophysiol. (2013) 87:19-27. doi: 10.1016/j.ijpsycho. 2012.10.01

Conflict of Interest Statement: The authors declare that the research was conducted in the absence of any commercial or financial relationships that could be construed as a potential conflict of interest.

Copyright (C) 2019 Léonard, Clément, Kuo and Manto. This is an open-access article distributed under the terms of the Creative Commons Attribution License (CC BY). The use, distribution or reproduction in other forums is permitted, provided the original author(s) and the copyright owner(s) are credited and that the original publication in this journal is cited, in accordance with accepted academic practice. No use, distribution or reproduction is permitted which does not comply with these terms. 